\title{
Mediación pedagógica en salud sexual y reproductiva, derechos sexuales y reproductivos dirigida a adolescentes ${ }^{1}$
}

Verónica Meza Arguedas ${ }^{2}$

Institución: Hogar Madre Redentor de Pérez Zeledón

\section{COMO CITAR}

Meza, V. (2016). Mediación pedagógica en salud sexual y reproductiva, derechos sexuales y reproductivos dirigida a adolescentes. Rev. Enfermería Actual en Costa Rica, 31, 1-16. DOI: http://dx.doi.org/10.15517/revenf.v0i31.24755

\section{RESUMEN}

Introducción. Al abordar el tema de la sexualidad desde el ámbito educativo, se han utilizado diversos métodos y procesos que, a lo largo de la historia, contribuyeron y facilitaron el desarrollo y la transmisión de conocimientos desde un sujeto o mediador, hacia una población determinada. Por ello es importante analizar la forma en cómo los temas de sexualidad se han transmitido a la población de adolescentes.. El objetivo fue desarrollar estrategias de mediación pedagógica en salud sexual, salud reproductiva, derechos sexuales y reproductivos dirigidas a adolescentes que viven en el Hogar Madre Redentor de Pérez Zeledón, 2013.

Método. Participó un grupo de adolescentes (15) entre 10 y 19 años, residentes del Hogar Madre Redentor de Pérez Zeledón. El estudio es diseño no experimental transeccional con un enfoque cuantitativo de tipo descriptivo. Resultado. Los resultados indican que utilizar métodos y técnicas pedagógicas interactivas, aunados al conocimiento, la experiencia y la práctica de un profesional en enfermería obstétrica, resultan muy beneficiosos, puesto que las participantes lograron comprender tanto los componentes de la adolescencia y la sexualidad, así como la importancia de mantener una vida sexual sana.

Conclusión. Es importante que el profesional en enfermería en obstetricia conozca y desarrolle técnicas educativas con el fin de facilitar un aprendizaje colaborativo sobre salud sexual, salud reproductiva, derechos sexuales y derechos reproductivos, principalmente porque el efecto que causan es seguro para el aprendizaje y la alfabetización sexual de diferentes poblaciones, especialmente aquellas representadas por adolescentes.

Palabras clave. Adolescentes, mediación-pedagógica, salud-sexual, salud-reproductiva, derechos-sexuales-yreproductivos.

\footnotetext{
${ }^{1}$ Fecha de recepción: 11 de febrero del 2016

Fecha de aceptación: 01 de junio del 2016

${ }^{2}$ Enfermera Ginecoobstetra. Trabajo independiente. Costa Rica. Correo electrónico: veromeza15@hotmail.com
} 


\title{
Pedagogical mediation in sexual and reproductive health, sexual and reproductive rights for adolescents ${ }^{1}$
}

\author{
Verónica Meza Arguedas ${ }^{2}$
}

Institution: Madre Redentor de Pérez Zeledón Home

\section{CITED}

Meza, V. (2016). Pedagogical mediation in sexual and reproductive health, sexual and reproductive rights for adolescents. Rev. Enfermería Actual en Costa Rica, 31, 1-16. DOI: http://dx.doi.org/10.15517/revenf.v0i31.24755

\begin{abstract}
Introduction. This article presents the results of the implementation of strategies for pedagogical mediation in Sexual Health, Reproductive Health, Sexual Rights and Reproductive Rights. The aim was to develop strategies for teaching mediation in Sexual Health, Reproductive Health, Sexual Rights and Reproductive Rights for adolescents living at home Madre Redentor de Pérez Zeledón, 2013.

Method. It involved a group of adolescents (15) between 10 and 19 years, residents of the Madre Redentor de Pérez Zeledón Home. Within the parameters of non-experimental design with a descriptive quantitative approach. A questionnaire, was previously validated prior to the implementation of mediation, in order to verify the pre- and post-knowledge of the participants on Sexual and Reproductive Health, The implementation of mediation was established in four basic units: 1. Adolescents, 2. Sexuality in adolescence, 3. Health and sexual and reproductive rights, 4. Self-assessment and self-knowledge.

Result. The results indicate that by using interactive teaching methods and techniques, coupled with the knowledge, experience and professional practice in midwifery, are very beneficial. Since the participants were able to understand both components of adolescence and sexuality, as well as the importance of maintaining a healthy sex life.

Conclusion. It is important that the nurse practitioner in obstetrics learns to develop educational techniques in order to facilitate collaborative learning about sexual health, reproductive health, sexual rights and reproductive rights.
\end{abstract}

Key words. Adolescents, pedagogical-mediation, reproductive-health, sexual-health, sexual-and-reproductiverights.

\footnotetext{
${ }^{1}$ Date of receipt: February 11, 2016

${ }^{2}$ Midwife. Independent work. Costa Rica. E-mail: veromeza15@hotmail.com
} 


\section{Mediação pedagógica em saúde sexual e reprodutiva, direitos sexuais e reprodutivos destinado a adolescentes ${ }^{1}$}

Instituição: Casa Madre Redentor de Pérez Zeledón

Verónica Meza Arguedas ${ }^{2}$

\section{CITAÇÃO}

Meza, V. (2016). Mediação pedagógica em saúde sexual e reprodutiva, direitos sexuais e reprodutivos destinado a adolescentes. Rev. Enfermería Actual en Costa Rica, 31, 1-16. DOI: http://dx.doi.org/10.15517/revenf.v0i31.24755

\section{RESUMO}

Introdução. Este artigo apresenta os resultados da implementação de estratégias de mediação pedagógica em Saúde Sexual, Saúde Reprodutiva, Direitos Sexuais e Direitos Reprodutivos. O objetivo foi desenvolver estratégias para ensinar a mediação em Saúde Sexual, Saúde Reprodutiva, Direitos Sexuais e Direitos Reprodutivos para adolescentes que vivem em casa Mãe Redentor Pérez Zeledón, 2013.

Método. Tratava-se de um grupo de adolescentes (15) entre 10 e 19 anos, residentes do Casa Mãe Redentor Perez Zeledón. Dentro dos parâmetros de design não experimental, com abordagem quantitativa descritiva. Um questionário, que é previamente, antes da implementação da mediação e posterior das mesmas aplicadas, a fim de verificar o pré e pós-conhecimento dos participantes sobre Saúde Sexual e Reprodutiva, foi utilizado lei Sexual e Reprodutiva para coleta de dados . A implementação da mediação foi estabelecida em quatro unidades básicas: 1 . Adolescentes, 2. sexualidade na adolescência, 3. saúde e direitos sexuais e reprodutivos, 4. Auto-avaliação e autoconhecimento.

Resultado. Os resultados indicam que, usando métodos de ensino interativos e técnicas, juntamente com o conhecimento, experiência e prática profissional em obstetrícia, são muito benéficas, uma vez, os participantes foram capazes de compreender ambos os componentes da adolescência e sexualidade, bem como a importância de manter uma vida sexual saudável.

Conclusão. É importante que a enfermeira em obstetrícia aprender e desenvolver técnicas de ensino, a fim de facilitar a aprendizagem colaborativa sobre a saúde sexual, saúde reprodutiva, direitos sexuais e direitos reprodutivos, principalmente porque o efeito causado por ele é seguro para a aprendizagem e literacia sexual de diferentes populações, especialmente aqueles representados por adolescentes.

Palavras chave. Adolescentes, mediação-pedagógica, saúde-sexual, saúde-reprodutiva, direitos-sexuais-ereprodutivos.

\footnotetext{
${ }^{1}$ Data de recebimento: 11 de fevereiro de 2016

${ }^{2}$ Enfermeira. Trabalho independente. Costa Rica. Correio eletrônico: veromeza15@hotmail.com
} 


\section{Revista Electrónica Enfermeria Actual en costa Rica}

\section{www.revenf.ucr.ac.cr}

\section{INTRODUCCIÓN}

Para abordar el tema de la sexualidad desde el ámbito educativo, se han utilizado diversos métodos y procesos que, a lo largo de la historia, contribuyeron y facilitaron el desarrollo y la transmisión de conocimientos desde un sujeto o mediador, hacia una población determinada. Por ello es importante analizar la forma en cómo los temas de sexualidad se han transmitido a la población de adolescentes.

Para el año 2006, en Valencia, España, se realizó un estudio denominado "Intereses, conducta sexual y comportamientos de riesgo para la salud sexual de escolares adolescentes participantes en un programa de educación sexual", basado en "describir las conductas sexuales más frecuentes en escolares adolescentes participantes en el programa, grado de información y conocimientos en sexualidad y sus fuentes; creencias, actitudes y mitos en sexualidad; intereses y demandas de información" (alenzuela, 2006). Los resultados del estudio enfatizan la necesidad de educar en materia sexual tanto a los adolescentes como a los padres de familia, por ser estos la principal fuente de información sexual para la población de jóvenes.

Por su parte en el año 2009, en España, se realizó un estudio titulado "Educación integral en sexualidad y anticoncepción para los/las jóvenes", el cual evidencia, según sus autores "el descenso de la edad en la que los jóvenes inician las relaciones sexuales, las actitudes cada vez más liberales, el mayor número de parejas y las prácticas sexuales de riesgo, los embarazos no deseados y las infecciones de transmisión sexual" (González, González y Sánchez, 2009, p.221). De acuerdo con los resultados derivados de la investigación, los autores concluyen que

La necesidad de una educación integral en sexualidad y anticoncepción dirigida a jóvenes españoles es una prioridad. Tanto las escuelas como las familias y los profesionales sanitarios, son fuentes de educación para la salud. Es necesario realizar este tipo de actividades comunitarias, siendo un instrumento para reducir los embarazos no deseados en jóvenes, las tasas de ITS y permitir que consigan una salud sexual y reproductiva plena (González, González y Sánchez, 2009, p.223).

A nivel internacional, principalmente en España, la educación sexual es una necesidad para los adolescentes, puesto que el desarrollo integral de ésta permite a la población minimizar riesgos y aumentar las posibilidades de mantener una salud sexual y reproductiva digna.

Por su parte en el continente americano, de igual forma se visualizan investigaciones en las que se concluyó que la utilización de diversos procesos educativos, para la educación sexual, resultan efectivos.

Por ejemplo, en 2004, se realizó en Santiago, Chile, una investigación, titulada "Embarazo en la adolescencia. Educación sexual y anticoncepción previa", el objetivo de la misma se basó, en "dar a conocer la relación previa entre la educación sexual y el uso de métodos anticonceptivos (MAC) en las adolescentes que se embarazan" (Díaz, Sugg y Valenzuela, 2004, p.79). Según, los autores 


\section{Revista Electrónica Enfermeria Actual en costa Rica}

Es clara la relación del embarazo en la adolescencia con una mayor morbi-mortalidad materna, mortalidad perinatal, aborto, problemas psicológicos en la madre... lo cual hace imprescindible tomar medidas de prevención y promoción de conductas sexuales responsables entre los adolescentes. Para esto, la educación sexual constituye un pilar fundamental (Díaz et al, 2004, p. 82)

En México, Gallegos (2005), publicó los resultados de su investigación, titulada "Intervención para reducir riesgo en conductas sexuales de adolescentes: un ensayo aleatorizado y controlado", la cual abordó el uso de métodos anticonceptivos y la promoción de la salud sexual. De acuerdo con el autor, el estudio demostró que "no hubo diferencia en las intenciones de los adolescentes de tener relaciones sexuales, pero sí mayor nivel de intenciones de usar condón y anticonceptivos". "La intervención conductual representa un importante esfuerzo en la promoción de conductas sexuales seguras en adolescentes mexicanos". (p.59); es decir, se fomentó una conducta de responsabilidad en el comportamiento sexual de las y los adolescentes participantes.

Por su parte en Colombia, de acuerdo con las publicaciones de García (2007), sobre su investigación, relacionada con la diversidad sexual en la escuela y las dinámicas pedagógicas necesarias para enfrentar la homofobia, el autor admite que se han generado mediaciones pedagógicas que dan auge a planes educativos sobre sexualidad.

En Costa Rica, en el año 2005, se realizó un Trabajo Final de Graduación para optar por el grado de Licenciatura en Enfermería, de la Universidad de Costa Rica, cuya investigación se basó en un programa en educación sexual, salud reproductiva, derechos sexuales y derechos reproductivos, con estudiantes universitarios de la sede Occidente de San Ramón, UCR (2005). El objetivo se fundamentó en el desarrollo de un programa, con enfoque de género sensitivo, basado en la afectividad, con el cual los jóvenes logren construir o complementar sus conocimientos, así como fomentar una sexualidad plena e integral para la toma acertada de decisiones.

Según conclusiones de la investigación mencionada, la educación sexual se ha convertido en un eje longitudinal en el desarrollo de la sexualidad adolescente, puesto que es imprescindible para fomentar una adecuada transmisión de conocimientos, acerca del tema y fomentar una salud sexual y reproductiva óptima.

En el año 2009, Brenes, Gómez y Quiñones desarrollan un programa educativo con énfasis en el autocuidado para la salud sexual, reproductiva y derechos sexuales y reproductivos, dirigido a un grupo de mujeres adultas de 18 a 23 años, en el Colegio Universitario de Cartago, entre cuyos hallazgos se destaca que el adecuado suministro de información sobre salud sexual y salud reproductiva permite a las adolescentes mantener prácticas adecuadas respecto de su sexualidad, lo cual incluye desde aspectos relacionados directamente con el individuo, como lo es el autocuidado, hasta la toma de decisiones que pueda afectar a terceros, como al evitar embarazos adolescentes. Por ello, los investigadores concluyen que

La importancia de la promoción de la salud y la prevención de la enfermedad, deben ser un campo en el cual los profesionales en enfermería se comprometan a expandir campos y abrir nuevos horizontes en la temática de la sexualidad holística (p. 212) 


\section{Revista Electrónica Enfermeria Actual en costa Rica}

Lo anterior quiere decir que la participación activa y conjunta de los profesionales en salud en el campo de la alfabetización de la adolescencia en materia de salud sexual y salud reproductiva, representa la oportunidad de brindar los servicios educativos adecuados y atinentes a un gran segmento de la población que presenta la mayor necesidad de conocimiento sobre el tema.

Luego, en el año 2010, se realizó una investigación para optar por el grado de Maestría en Enfermería Ginecológica, Obstétrica y Perinatal en la Universidad de Costa Rica, denominada "Programa educativo en salud sexual dirigido a los y las jóvenes de la sección 9-1 y 9-4 del Liceo San José, de Alajuela, en el periodo octubrenoviembre, del año 2010", en la cual

se evidencia la necesidad educativa de los jóvenes adolescentes en relación con el aspecto biológico, social, emocional y espiritual de la salud sexual.

La ejecución de dicho programa es relevante para enfermería, ya que contribuye un espacio valioso para dar a conocer los amplios aportes que puede brindar esta disciplina en cuanto a la salud y prevención de la enfermedad, en estos grupos de edad fomentando la construcción de los conocimientos (Carballo, Granados \& Sanabria, 2010, p.104-108).

Las investigaciones citadas evidencian la importancia de la educación sexual en la adolescencia, sin embargo, en las estrategias educativas utilizadas en dichas intervenciones no abarcan en su totalidad el tema de salud sexual y reproductiva, derechos sexuales y reproductivos, sino que se abordan de manera parcial, principalmente al relacionarlos con salud sexual, autocuidado, consejerías sobre salud, entre otros.

La salud sexual y reproductiva, derechos sexuales y reproductivos, así como la educación sexual, son temas que han tomado auge en los últimos años. Muchos son los aspectos tratados al respecto entre los que se puede mencionar la Conferencia Internacional sobre la Población y el Desarrollo, CIPD (1994), realizada en el Cairo, en la que se establecen las bases para el correcto accionar con el tema de salud y educación sexual, así como los siguientes objetivos, los cuales se encuentran vigentes en las conferencias internacionales de los años 2004 y 2009:

Asegurar el acceso a información amplia y fáctica y a una gama completa de servicios de salud reproductiva, incluida la planificación de la familia, que sean accesibles, asequibles y aceptables para todos los usuarios;

Propiciar y apoyar decisiones responsables y voluntarias sobre la procreación y sobre métodos libremente elegidos de planificación de la familia, así como sobre otros métodos que puedan elegirse para la regulación de la fecundidad que no estén legalmente prohibidos, y asegurar el acceso a la información, la educación y los medios necesarios;

Atender a las necesidades cambiantes en materia de salud reproductiva durante todo el ciclo vital, de un modo que respete la diversidad de circunstancias de las comunidades locales (Naciones Unidas 1995, p. 38),

En torno al tema, Florez y Soto (2008) agregan que "el conocimiento de temas relacionados con la salud sexual y salud reproductiva indica vacíos importantes en todos los países en cuanto a la fisiología de la reproducción, y además un desconocimiento en los países de transición rezagada..” (p.30), lo cual pone en 


\section{Revista Electrónica Enfermeria Actual en costa Rica}

manifiesto una falta de educación sexual en la población, principalmente en países subdesarrollados, puesto que los programas educativos que se implementan tanto en el exterior como a nivel nacional, en materia de sexualidad, solo atienden a objetivos meramente fisiológicos, es decir, son simplemente informativos y tratan aspectos generales de la salud sexual y salud reproductiva en la adolescencia. Sin embargo, Costa Rica, no cuenta con servicios de salud dirigidos principalmente a los adolescentes, al punto de que se ha generado un desfase entre la necesidad y la capacidad técnica del sector salud, para brindar servicios oportunos a los adolescentes en materia de educación y cuidados de la sexualidad del segmento.

Por ello, para la mayoría de los adolescentes, tales temas son conocidos, pero poco comprendidos, por la falta de información y educación sexual, mas todavía no existen estudios que midan el impacto del programa del Ministerio de Educación en la población destino, lo cual es afirmado por Mosquera y Mateus, para quienes "los jóvenes tienen conocimientos inapropiados acerca de los Métodos de Planificación Familiar y esto se refleja en sus prácticas y sugiere que en necesario reforzar los procesos de educación y comunicación con los (as) jóvenes y sus padres" (2003, p. 206).

Por otra parte, las investigaciones realizadas por estudiantes universitarios, basados en programas educativos para la salud y derechos sexuales no son suficientes para la capacitación de la población de adolescentes de todo el país, principalmente por la gran cantidad de poblaciones vulnerables. Debido a ello, se vuelve necesaria la inclusión de mediaciones pedagógicas dentro de ciertos sectores sociales, principalmente por el limitado acceso a la correcta información y por el derecho de los jóvenes a tener una educación sexual adecuada, impartida a través de metodologías educativas efectivas realizadas por personal de salud calificado, en este caso, los profesionales especializados en la maestría en enfermería ginecológica, obstétrica y perinatal, máxime considerando que

La mediación pedagógica establece un tipo de dirección del aprendizaje que no es ni directa, ni frontal. Todo lo contrario indirecta y con la participación activa de los implicados en el proceso. Es decir, propiciando la interacción y la interactividad de los alumnos en "clase", presencial o a distancia (Lima, 2005, p. 8).

Es claro entonces que el uso de diferentes estrategias de mediación pedagógica, permitirá a dicho profesional fomentar la aplicación de conocimientos vinculados con la sexualidad y su importancia, relacionando el proceso de enseñanza y aprendizaje con las situaciones reales suscitadas en la sociedad actual, de manera que generen un aprendizaje significativo en los adolescentes y propicie el desarrollo de una sexualidad plena, madura y responsable, permitiendo al adolescente una mejor comunicación con el resto de la sociedad.

Dado lo anterior, que un profesional en enfermería obstétrica, realice este tipo de intervenciones educativas, es de suma importancia para la población de adolescentes, lo cual queda establecido en el Reglamento de Enfermería Ginecobstetricia y Perinatal de Costa Rica, el cual indica como competencia del profesional,:

Fortalecer la atención integral de Enfermería Ginecobstétrica y Perinatal en la promoción de la salud sexual y reproductiva, derechos sexuales y reproductivos de hombres y mujeres en los ciclos de vida (niñez, adolescencia, juventud, adultez y adultez mayor) en el primer nivel de atención en los escenarios de salud establecidos (Colegio de enfermeras de Costa Rica, 2005). 


\section{Revista Electrónica Enfermeria Actual en costa Rica}

Es indiscutible cuán relevante es emplear estrategias de mediación pedagógica para brindar educación en salud sexual y reproductiva en la población adolescente. De hecho, como se expuso, esta actividad es parte de las labores que un profesional en enfermería ginecológica, obstétrica y perinatal debe realizar, tanto a nivel público como privado.

El objetivo de esta investigación aplicada fue desarrollar estrategias de mediación pedagógica en salud sexual, salud reproductiva, derechos sexuales y derechos reproductivos dirigidas a las adolescentes que viven en el Hogar Madre Redentor de Pérez Zeledón, 2013.

\section{MATERIALES Y MÉTODOS}

En el presente estudio, la población fue constituida por 15 adolescentes que residen en el hogar. Se trabajó solamente con 15:, 9 entre 10 y 14 años y 6 entre 15 y 19 años, debido a que una de las jóvenes decidió no ser partícipe de la mediación y abandonó el grupo desde la primera sesión.

La población se considera homogénea puesto que las jóvenes poseen características similares según los rangos de edades, comparten experiencias comunes dentro del hogar y además viven el periodo de la adolescencia, el cual difiere de un individuo a otro, pero ninguno queda exento de vivirla.

Esta investigación se diseñó como no experimental, transeccional: no experimental porque no se pueden manipular las variables, los datos a reunir se obtendrán de la población de adolescentes del Hogar Madre Redentor de Pérez Zeledón, y transeccional, ya que la recolección de datos se realizó en un solo periodo de tiempo. Además, como menciona Hernández, "La investigación no experimental es la que se realiza sin manipular deliberadamente variables; lo que se hace en este tipo de investigación es observar fenómenos tal y como se dan en su contexto natural, para después analizarlos" (Hernández, Fernández y Baptista, 2010, p. 149).

Asimismo, la investigación obedece al tipo descriptivo, el cual tiene como objetivo describir las variables o categorías que se estudian, es decir, "indagan la incidencia de las modalidades, categorías o niveles de una o más variables en la población, son estudios puramente descriptivos" (Hernández et al, 2010, p. 152).

El instrumento utilizado para realizar el diagnóstico y la evaluación de las estrategias de mediación pedagógica es el cuestionario, formado por 26 preguntas, divididas en cuatro apartados explicados a continuación:

Apartado 1: Información general: Mediante preguntas cerradas se obtiene información sobre el sexo, la edad, la nacionalidad y la escolaridad de las participantes.

Apartado 2: Aspectos cognitivos sobre adolescencia y sexualidad: Mediante preguntas cerradas y abiertas se les interroga sobre aspectos relacionados con adolescencia, sexo, identidad de género, sexualidad y genitalidad.

Apartado 3: Aspectos cognitivos relacionados con salud y derechos sexuales y reproductivos: Se indaga con la utilización de preguntas cerradas y abiertas conceptos como: relaciones sexuales, métodos anticonceptivos, ITS, salud y derechos sexuales, otros. 


\section{Revista Electrónica Enfermeria Actual en costa Rica}

Apartado 4: Educación sexual: Utiliza preguntas cerradas para indagar el conocimiento de las participantes sobre el concepto educación sexual y la escogencia de temas poco conocidos o desconocidos sobre los que deseen obtener información.

El instrumento descrito fue validado por 10 jueces quienes emitieron criterios respecto del formato y el fondo. Se consideró las recomendaciones emitadas por los jueces y se elaboró una versión final del instrumento.

Este instrumento fue aplicado antes y posterior a la implementación de la mediación.

\section{Consideraciones éticas}

En el planteamiento de cualquier investigación en la que se trabaje con personas, es trascendental tomar en cuenta los aspectos éticos de la investigación con el fin de proteger la integridad de los participantes, tal como el consentimiento informado, en el cual según Galeano, se debe aclarar los aspectos éticos por considerar: autonomía, beneficencia, no maleficencia, etc. Su uso parte de que

Los informantes o participantes en el proceso investigativo deben ser consultados y lograr acuerdos con el investigador sobre la utilización de técnicas de registro no intrusivas (notas de campo), intrusivas como grabadora, videograbadora, cámara fotográfica. Igualmente tienen derecho a conocer que información va ser registrada, con que propósitos, si va ser compartida con otros investigadores y a que publico va llegar. (2004, p. 74)

Además del consentimiento informado, se consideró aspectos como la confidencialidad y el anonimato de las participantes; por lo que en el análisis de los resultados, no figuran nombres ni características específicas de cada una de las adolescentes. Se contó con los respectivos permisos de la institución.

\section{RESULTADOS}

\section{Etapa diagnóstica}

En cuanto a las características sociodemográficas de las participantes, las 15 participantes son de nacionalidad costarricense, con edades entre los 10 y los 19 años. En lo que respecta a la escolaridad, 9 de ellas tienen primaria incompleta y 6, secundaria incompleta. Todas las residentes son solteras. En relación con la permanencia, 7 de ellas tienen 1 año de residencia, 3 han permanecido en el lugar durante 2 años y las 5 restantes han habitado por más de 2 años.

\section{Conocimiento de las adolescentes sobre adolescencia y sexualidad}

En este apartado se obtuvo que 9 participantes conocen el concepto de adolescencia, mientras que 6 no se familiarizan con este; por ende, se afirma que las participantes no conciben la adolescencia como un proceso integral y que el conocimiento incide en las decisiones sobre la sexualidad del adolescente. 


\section{Revista Electrónica Enfermeria Actual en costa Rica}

En lo que respecta al concepto de sexualidad, las 15 participantes desconocen al respecto: para ellas, la sexualidad se encuentra representada por aspectos inherentes al término, pero de forma individualizada.

\section{Conocimiento de las adolescentes sobre salud sexual y reproductiva derechos sexuales y reproductivos}

Con base en el concepto de relaciones sexuales, 4 de las adolescentes aseguran saber qué son, mientras que 11 , desconocen al respecto; por consiguiente, la situación es de un total desconocimiento, por cuanto confunden el término con los conceptos de salud sexual y sexualidad.

Por su parte, sobre el conocimiento de métodos anticonceptivos, 4 adolescentes poseen conocimiento sobre qué son y para qué se utilizan los métodos anticonceptivos, las 11 restantes desconocen la información, motivo por el que se intuye que el conocimiento que poseen es meramente superficial, debido a que desconocen los diferentes tipos de anticonceptivos, así como el uso y finalidad de cada uno.

En lo referente a salud sexual, todas las participantes poseen un concepto focalizado en un solo aspecto de la salud sexual, por lo que es importante rescatar que la salud sexual representa diversos aspectos, pero de forma aislada, así como fomentar la salud sexual ampliamente dentro de la mediación.

Una situación similar se presenta con los derechos sexuales y reproductivos, donde las adolescentes, visualizan los derechos sexuales y reproductivos como fuentes de protección, lo cual redunda en una desvinculación de la autonomía ligada al término.

Como último aspecto del apartado de diagnóstico, en cuanto al concepto y alcance de la educación sexual, 3 de las consultadas entienden correctamente lo que abarca la educación sexual, mientras que las 12 restantes no entienden sobre el tema: para estas la educación sexual obedece meramente al ámbito biológico del ser humano.

\section{Resultados de la etapa de diseño de la mediación}

El diseño de la mediación se encuentra constituido por introducción - justificación - descripción de la mediación, así como por las diferentes unidades, con sus respectivos temas y matrices educativas, las cuales se citan a continuación con su respectivo nombre y temáticas.

\section{UNIDAD 1: Yo como adolescente}

Temas por tratar: Adolescencia. Cambios físicos y emocionales en la adolescencia. Factores protectores.

\section{UNIDAD 2: Sexualidad}

Temas por tratar: Sexualidad. Genitalidad. Género. Mitos y Creencias sobre la sexualidad.

\section{UNIDAD 3: Salud sexual y reproductiva, derechos sexuales y reproductivos}

Temas por tratar: Salud sexual y Salud reproductiva. Derechos sexuales y Derechos reproductivos. Relaciones sexuales. Infecciones de trasmisión sexual. Anticoncepción. Maternidad y paternidad. 


\section{Revista Electrónica Enfermeria Actual en costa Rica}

\section{www.revenf.ucr.ac.cr}

\section{UNIDAD 4: Factores protectores en la adolescencia \\ Temas a tratar: Autoconocimiento. Autoevaluación.}

Formato de matriz utilizado para las diferentes clases

Mediación Pedagógica en Salud Sexual, Salud Reproductiva, Derechos Sexuales y Derechos Reproductivos dirigidos a un grupo de adolescentes entre 10 y 19 años ubicadas en el Hogar Madre Redentor de Pérez Zeledón, 2013. Matriz Educativa: \# Sesión

UNIDAD DE ESTUDIO:

TIEMPO ESTIMADO:

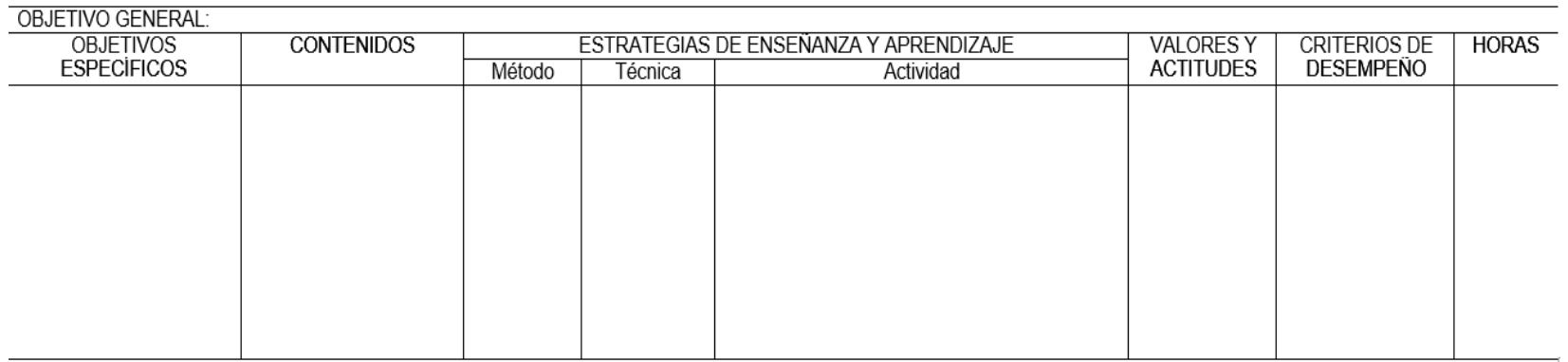

\section{Resultados de la etapa de implementación de la mediación}

La etapa de implementación desarrolla las 4 unidades pedagógicas mencionadas en el diseño de la mediación.

Dentro de la unidad 1 "Yo como adolescente" se transmitió a las participantes conceptos atinentes a la adolescencia (cambios, físicos y emocionales, factores protectores), como base introductoria a temas meramente sobre sexualidad. Las técnicas utilizadas fueron conferencia, lluvia de ideas, collage, preguntas y respuestas, de las que se obtuvo resultados positivos, pues se emprendió una plática con mucha confianza. Al finalizar esta unidad, las jóvenes habían adquirido conocimientos sobre conceptos básicos asociados a la adolescencia.

Para la unidad 2 "Sexualidad en la adolescencia", se enfatizó en dotar a las jóvenes de conceptos acertados y verdaderos sobre sexualidad, género, identidad de género, genitalidad, diferencias entre los conceptos, así como la aclaración de los mitos y creencias sobre el tema.

Se utilizó la técnica conferencia interactiva, para la explicación de diferentes términos, un taller para la diferenciación entre los términos así como preguntas y respuestas seguido de una lección desarrollada por la mediadora. Al finalizar, las adolescentes tenían conceptos claros sobre sexualidad y sus componentes, además se logró evacuar las dudas sobre mitos y creencias de la sexualidad.

En la unidad 3 "Salud Sexual y Reproductiva Derechos Sexuales y Reproductivos", los tópicos fueron desarrollados mediantes estrategias que acercaron a las participantes a la realidad, puesto que se utilizaron tanto los videoforos sobre salud sexual y reproductiva, derechos sexuales y reproductivos, así como la técnica conferencia y conferencia interactiva, para explicar a las participantes sobre las relaciones sexuales, infecciones de transmisión sexual, métodos anticonceptivos, y el derecho a la salud y a la educación sexual que posee este 


\section{Revista Electrónica Enfermeria Actual en costa Rica}

segmento de la población a nivel nacional e internacional. Al finalizar esta unidad, se demostró el conocimiento adquirido a lo largo de la mediación sobre la salud sexual y reproductiva y derechos sexuales y reproductivos y las formas de prevenir situaciones de riesgo. Se evidenció el alcance de los objetivos, puesto que las jóvenes lograron conocer de forma general y específica los aspectos que engloban la salud sexual, salud reproductiva, los derechos sexuales y reproductivos, es decir, desde lo que concierne a las ITS, anticoncepción humana, hasta la importancia de abordar y comprender este tipo de temáticas que ayudan a los adolescentes en el desarrollo de una vida sexual plena.

Luego, en la unidad 4 "Factores protectores en la adolescencia", se utilizó temáticas atinentes al autonocimiento y la autoevaluación de las participantes, mediante el desarrollo de talleres en los que realizaron un autorretrato y una evaluación de sí. El alcance de la temática deriva en la reflexión sobre la importancia de mantener una salud sexual y reproductiva, y defender sus derechos en cuanto al mismo tema.

\section{Resultados etapa de evaluación de la mediación}

Tabla 1. Hogar Madre Redentor de Pérez Zeledón. Distribución de la comparación de resultados del diagnóstico vs evaluación según temas desarrollados, 2013.

\begin{tabular}{r|c|c|c|c|}
\hline \multirow{2}{*}{$\begin{array}{c}\text { Tema } \\
\text { Conocimiento sobre: }\end{array}$} & \multicolumn{2}{|c|}{ Diagnóstico } & \multicolumn{2}{c}{ Evaluación } \\
\cline { 2 - 5 } & Conoce & No conoce & Conoce & No conoce \\
\hline Adolescencia & 9 & 6 & 11 & 4 \\
\hline Sexualidad & 0 & 15 & 9 & 6 \\
\hline Relaciones sexuales & 4 & 11 & 12 & 3 \\
\hline Métodos anticonceptivos & 4 & 11 & 15 & 0 \\
\hline Salud sexual y reproductiva & 0 & 15 & 15 & 0 \\
\hline Derechos sexuales y reproductivos & 0 & 15 & 15 & 0 \\
\hline
\end{tabular}

Respecto del conocimiento sobre adolescencia, las participantes que erraron en la respuesta, continúan confundiendo el término con la definición de salud sexual. Sin embargo ya no lo confunden con el concepto de genitalidad, como se demostró en el diagnóstico.

En lo referente al conocimiento sobre sexualidad, las jóvenes que aún no lograron comprender el concepto, siguen conceptualizando la sexualidad, como genitalidad, género y relaciones sexuales.

En el conocimiento respecto de las relaciones sexuales, las tres participantes que no lograron comprender el término, y aún lo continúan confundiendo con el concepto de sexualidad se debe a que

1. Pertenecen a la etapa temprana de la adolescencia.

2. Se encontraban cursando la primaria. 


\section{Revista Electrónica Enfermeria Actual en costa Rica}

La mediación pedagógica permitió a las adolescentes, fortalecer y/o adquirir conocimiento sobre temas relacionados con su salud sexual.

Dentro de estos ítems, se demostró que la implementación de la mediación pedagógica, permitió a las adolescentes, fortalecer y/o adquirir conocimiento sobre temas relacionados con su salud sexual y reproductiva, derechos sexuales y reproductivos.

Con los resultados anteriores se denota que la educación sexual, desarrollada a través de estrategias de mediación pedagógica, se convierte en una herramienta, que les proporcionará las pautas necesarias para el desarrollo de una vida sexual sana.

\section{DISCUSIÓN}

La implementación de estrategias educativas, como la mediación pedagógica, con poblaciones adolescentes, para tratar o bien fomentar el aprendizaje y/o la capacitación en temáticas referidas a salud sexual y reproductiva, derechos sexuales y reproductivos, se constituyen en un importante aporte de alfabetización de este segmento de la población con una marcada efectividad. Lo anterior se afirma por cuanto se demostró en la presente investigación, que las prácticas pedagógicas con diversos segmentos de la sociedad, desde el apoyo teórico práctico de un profesional en enfermería ginecoobstétrica y perinatal permiten que los participantes adquieran y/o fortalezcan conocimientos que les faciliten mantener una vida sexual sana.

Cabe recalcar que es necesario un proceso holístico donde se combinen etapas de diagnóstico, diseño, implementación y evaluación de la estrategia educativa que se utilice, con el fin de generar un procedimiento óptimo, adecuado y en función de las necesidades que la población presente.

El abordaje desde esta dimensión permite constatar la efectividad de la implementación y, por ende, generar las recomendaciones necesarias que permitan minimizar el factor error en investigaciones posteriores.

La alfabetización del segmento de adolescentes y demás sectores de la sociedad, en materia de salud sexual y reproductiva, derechos sexuales y reproductivos se constituye actualmente en uno de los retos del milenio, involucrándose en ello la Organización Mundial de la Salud, la Organización Panamericana de la Salud y la Asociación Mundial para la Salud Sexual, entre otros, los cuales de forma conjunta buscan gestionar mecanismos para alcanzar tal iniciativa, por tanto, el sector salud, al contar con el personal idóneo por su nivel de capacitación en el tema, debe convertirse en uno de los principales aliados en el alcance de los objetivos propuestos.

Ante tal realidad, Costa Rica presenta la Política Nacional de Enfermería, que indica la responsabilidad de este profesional dentro del "Fortalecimiento de un proceso de aprendizaje significativo en educación sexual y reproductiva, derechos sexuales-reproductivos en la niñez y adolescencia desde una perspectiva género sensitivo" (p.28), puesto que se cuenta con los recursos necesarios para alcanzar las metas propuesta en lo que a salud sexual compete. 


\section{Revista Electrónica Enfermeria Actual en costa Rica}

Es evidente la necesidad educativa sobre sexualidad de las adolescentes del Hogar Madre Redentor de Pérez Zeledón, en lo concerniente a la salud sexual, salud reproductiva, derechos sexuales y derechos reproductivos principalmente por el poco acceso a información relacionada con esta y con los derechos sexuales y reproductivos. De igual manera, que la población base de la investigación, carezca de un núcleo familiar, que la oriente y respalde en la toma de decisiones sobre su salud sexual, dificulta el grado de confianza en sí, por ser la familia la base fundamental sobre la que se forma y desarrolla el adolescente.

El grupo de iguales caracterizado por el compañerismo permite el adecuado desarrollo de las técnicas y actividades concernientes a la implementación de la mediación: en este caso, las técnicas y actividades interactivas, aunados a una actitud positiva, permitieron generar el vínculo de confianza necesario, para conocer las opiniones reales de la población investigada y para desarrollar con efectividad la mediación.

Es necesario que el profesional en enfermería ginecobstétrica y perinatal desarrolle mediaciones pedagógicas sobre salud sexual y reproductiva, derechos sexuales y reproductivos, dirigidas a poblaciones adolescentes, principalmente porque su preparación tanto académica como práctica permite y facilita la trasmisión de conocimientos a los participantes, al fortalecer de esta manera su entendimiento sobre el tema y orientar adecuadamente sus decisiones.

Asimismo, es importante rescatar que el trabajo conjunto de un profesional en enfermería y un profesional en psicología, facilita el desarrollo de las mediaciones, puesto que el conocimiento de ambos es vital tanto para la confección y aplicación de los instrumentos, como para la implementación de diversas estrategias y técnicas dentro del grupo participante.

\section{CONCLUSIÓN}

Es importante que el profesional en enfermería en obstetricia conozca y desarrolle técnicas educativas con el fin de facilitar un aprendizaje colaborativo sobre salud sexual, salud reproductiva, derechos sexuales y derechos reproductivos, principalmente porque el efecto que causan los mismos es seguro para el aprendizaje y la alfabetización sexual de diferentes poblaciones, especialmente aquellas representadas por adolescentes.

\section{Conflicto de intereses.}

La autora declara que no existe conflicto de intereses para el desarrollo de esta investigación. 


\section{Revista Electrónica Enfermeria Actual en costa Rica}

\section{REFERENCIAS BIBLIOGRÁFICAS}

Brenes, T., Gómez L., Quiñones, E. (2009). Programa educativo con énfasis en el autocuidado para la salud sexual, reproductiva y derechos sexuales y reproductivos, dirigido a un grupo de mujeres adultas de 18 a 23 años, en el Colegio Universitario de Cartago (C.U.C.), 2009. Práctica Dirigida para optar por el grado de Licenciatura de la Universidad de Costa Rica.

Carballo, I., Granados, M. y Sanabria, MT. (2010). Programa educativo en salud sexual dirigido a los y las jóvenes de la sección 9-1 y 9-4 del Liceo San José, de Alajuela, en el periodo octubre-noviembre, 2010. Tesis de Licenciatura. Escuela de Enfermería. Universidad de Costa Rica, San José

Colegio de Enfermeras de Costa Rica. (2005). Reglamento de Enfermería Ginecoobstétrica y Perinatal de Costa Rica. Reglamento 1565 de 7-1-2005. San José, Costa Rica: La Gaceta N${ }^{\circ}$.

Consejo Nacional de la Política Pública de la Persona Joven. (2008). Primera Encuesta Nacional de Juventud. la ed. UNFPA, Costa Rica.

Díaz, A., Sugg, C., Valenzuela, M. (2004). Embarazo en la adolescencia. Educación sexual y anticoncepción previa. Revista Sogia. 3, 79-83.

Flores, C., Soto, V. (2008). El estado de la salud sexual y reproductiva en América Latina y el Caribe: Una visión global. New York, EE.UU.

Fondo de las Naciones Unidas para la Infancia Unicef (2011). Informe Anual de Unicef, 2011. Unicef. División de Comunicaciones. New York, EE.UU.

Fondo de Población de las Naciones Unidas (UNFPA). (2003). Programa Nacional de Educación para la Sexualidad y Construcción de la Ciudadanía. Colombia.

Galeano, ME. (2004). Diseño de proyectos en la investigación cualitativa. Medellín: Universidad EAFIT.

Gallegos, E., Loveland, C., Ronis, D., Villarruel, M., Zhou, Y, (2008). Intervención para reducir riesgo en conductas sexuales de adolescentes: un ensayo aleatorizado y controlado. Recuperado el 3 de febrero de 2014, de http://www.scielo.org.mx/scielo.php?script=sci_arttext\&pid=S0036-36342008000100012.

García, C. (2007). Programa: Diversidad sexual en la escuela. Dinámicas pedagógicas para enfrentar la homofobia. Bogotá, Colombia.

González, A, Gonzales, C, Sánchez J. (2009). Educación integral en sexualidad y anticoncepción para los/las jóvenes. Revista Enfermería Clínica, 19, 221-224. 


\section{Revista Electrónica Enfermeria Actual en costa Rica}

Hernández, Fernández y Baptista. (2010) Metodología de la investigación. Quinta edición. México D. F.: Editorial McGrawHill.

Mosquera, J. y Mateus, JC. (2003) Conocimientos, actitudes y prácticas sobre métodos de planificación familiar, VIH-SIDA y el uso de los medios de comunicación en jóvenes. Rev. Colombia Médica 34 (4) 206-212. Recuperado de: http://colombiamedica.univalle.edu.co/index.php/comedica/article/view/275/278

Naciones Unidas (1995) Informe de la Conferencia Internacional sobre la población y el desarrollo (1994). El Cairo: UNPFA. Recuperado de UNPFA.org.

Lima, S. (s.f.) La mediación pedagógica con uso de las tecnologías de la información y comunicación (TIC) Instituto Superior Pedagógico Enrique José Varona. La Habana, Cuba. Recuperado de: http://www.unibe.edu.ec/index.php/documentacion-didactica/-8/105-ticmediacionpedagogica/file

Palenzuela, A. (2006). Intereses, conducta sexual y comportamientos de riesgo para la salud sexual de escolares adolescentes participantes en un programa de educación sexual. Revista Análisis y Modificación de Conducta, 32. $\mathrm{N}^{\circ} 144$. 